Received: 2016.11 .28

Accepted: 2016.12 .30

Published: 2017.09.15

Authors' Contribution:
A Study Design
B Data Collection
C Statistical Analysis
D Data Interpretation
E Manuscript Preparation
F Literature Search
G Funds Collection

\section{Extremely Rare Case of Bilateral Pure Primary Non-Gestational Ovarian Choriocarcinoma}

\author{
Moinullah Syed ${ }^{\text {ABGDEF, }}$, Shefali Meshram ${ }^{\text {BGE, }}$, Pooja Deshpande ${ }^{\text {ABDD }}$, Bikash Parida ${ }^{\text {IB }}$ \\ Department of Radiodiagnosis, Byramjee Jeejeebhoy Government Medical College and Sassoon General Hospitals, Poona, \\ Maharashtra, India
}

Author's address: Moinullah Syed, Department of Radiodiagnosis, Byramjee Jeejeebhoy Government Medical College and Sassoon General Hospitals, Poona, Maharashtra, India, e-mail: moin.bmc@gmail.com

\begin{tabular}{|c|c|}
\hline & Summary \\
\hline Background: & $\begin{array}{l}\text { Germ cell tumors of the ovary constitute less than one percent of ovarian tumors worldwide. } \\
\text { Choriocarcinoma arising de novo from the ovary is very rare and only occasionally reported in } \\
\text { the literature. Herein, we report a case of bilateral non-gestational pure primary ovarian } \\
\text { choriocarcinoma that was confirmed by beta human chorionic gonadotropin ( } \beta \text {-HCG) levels and } \\
\text { histopathology. }\end{array}$ \\
\hline Case Report: & $\begin{array}{l}\text { Our case is of a middle-aged multiparous female who presented with amenorrhea for three } \\
\text { months. She underwent an evaluation with ultrasound (US), computed tomography (CT), and } \\
\text { magnetic resonance imaging (MRI), which revealed bilateral bulky solid adnexal masses. Based } \\
\text { on an increased blood level of the beta human chorionic gonadotropin and a histopathological } \\
\text { examination, the diagnosis of bilateral non-gestational pure primary ovarian choriocarcinoma was } \\
\text { made. }\end{array}$ \\
\hline Conclusions: & $\begin{array}{l}\text { The imaging findings were found to be specific for bilateral non-gestational pure primary ovarian } \\
\text { choriocarcinoma. }\end{array}$ \\
\hline eSH Keywords: & Choriocarcinoma • Chorionic Gonadotropin • Neoplasms, Germ Cell and Embryonal • Ovarian Neoplasms \\
\hline e: & /www.polradiol.com/abstract/index/idArt/902578 \\
\hline
\end{tabular}

\section{Background}

Of all germ cell tumors of the ovary, choriocarcinomas are the rarest. They can be seen in the ovary either due to metastases from uterine choriocarcinoma or due to primary tumors arising with or without an antecedent cause, of which the latter is a rare occurrence. In this case report, we describe the imaging findings of bilateral pure primary ovarian choriocarcinoma with serological and histopathological correlates.

\section{Case Report}

A thirty-eight-year-old multiparous female patient presented to our institution with a history of amenorrhea for three months. She had her last full-term uneventful pregnancy five years earlier. Her urine pregnancy test was positive. She was referred to our department for ultrasound (US) to confirm pregnancy. No obvious intra-uterine gestational sac was seen. However, iso-to-hyperechoic bulky solid mass lesions were noted in bilateral adnexa (Figure 1A, 1B). Neither of the ovaries was separately visualized. Cystic spaces with dense moving echoes were seen in the right adnexal lesion, possibly due to hemorrhage. On color Doppler, both lesions showed significant vascularity (Figure 2A, 2B). The plasma $\beta$-HCG titers were found to be three hundred thousand milli-international units per milliliter $(300,000 \mathrm{mIU} / \mathrm{mL})$. On plain and contrast-enhanced computed tomography (CT), bilateral bulky hypodense ovarian masses were seen. Few hyperdense areas were noted in the right ovarian lesion (Figures 3,4), suggestive of hemorrhage. Both masses showed moderate heterogeneous enhancement (Figure 5). Multiple dilated veins were noted adjacent to the tumors, signifying a vascular nature. Plain and contrast-enhanced magnetic resonance imaging (MRI) showed these lesions as iso- to slightly hyperintense on TIW images, and heterogeneously hyperintense on T2W and short tau inversion recovery (STIR) sequences (Figures 6-8). Areas of hyperintensity were seen in both ovarian masses on TlW images that showed blooming 


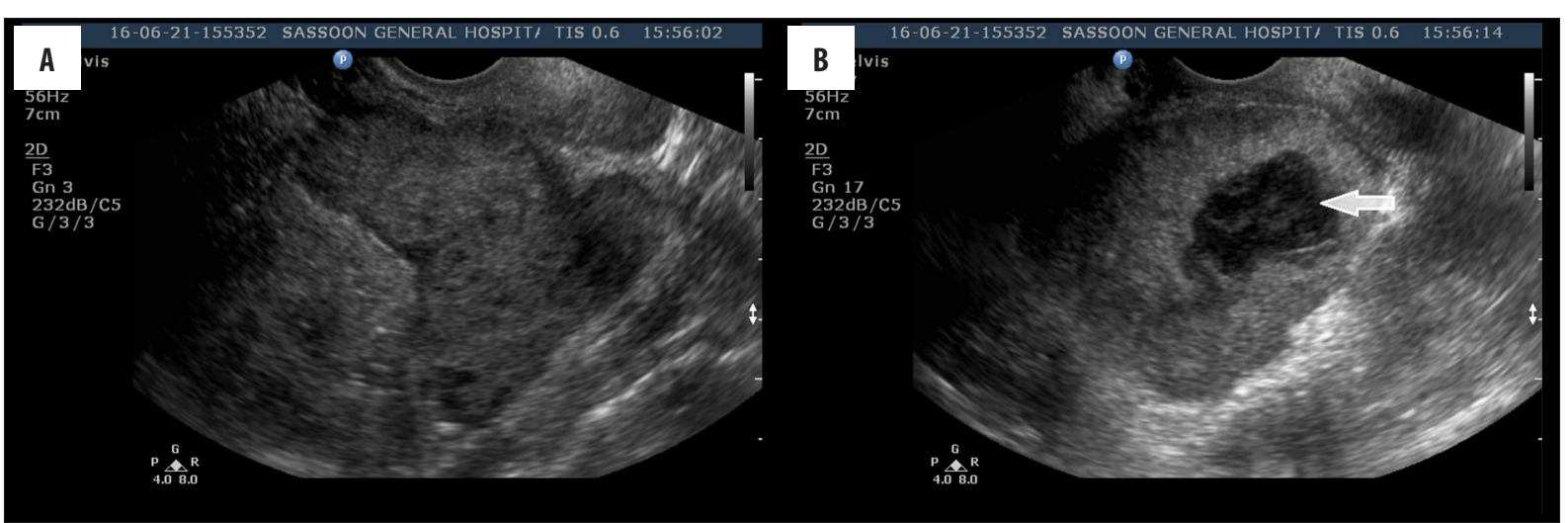

Figure 1. (A, B) Ultrasound images show bilateral adnexal mass lesions with hemorrhagic changes in the right mass (arrow)

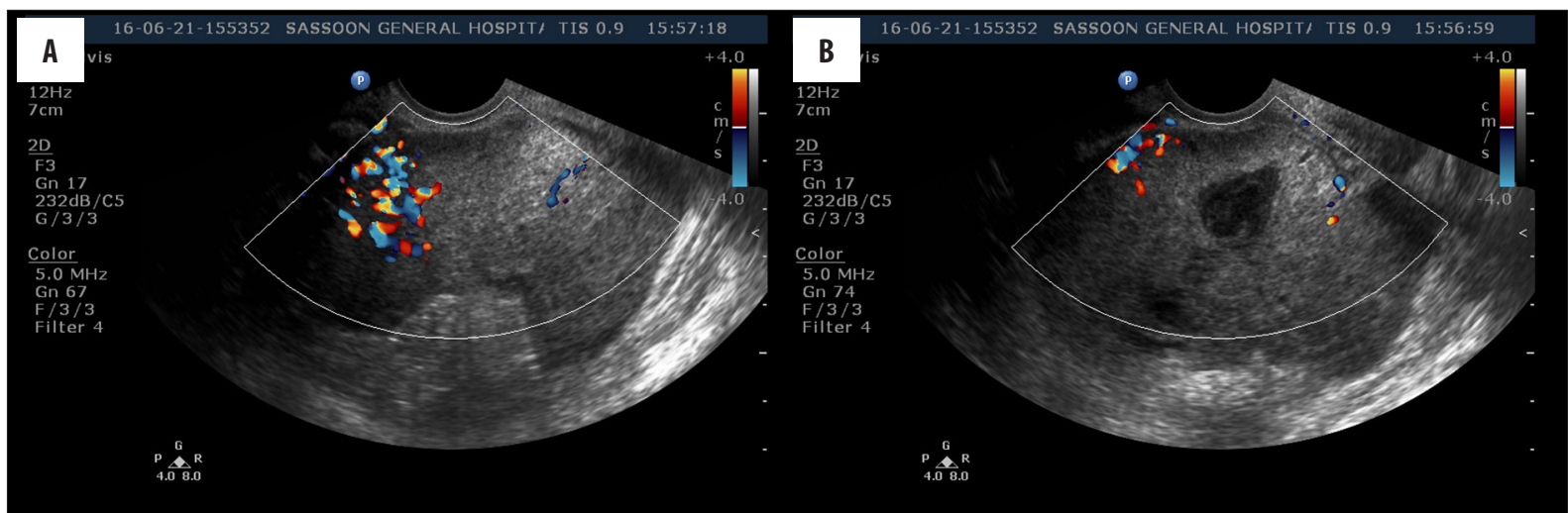

Figure 2. (A, B) Color Doppler images of same lesions show a vascular nature of the masses.

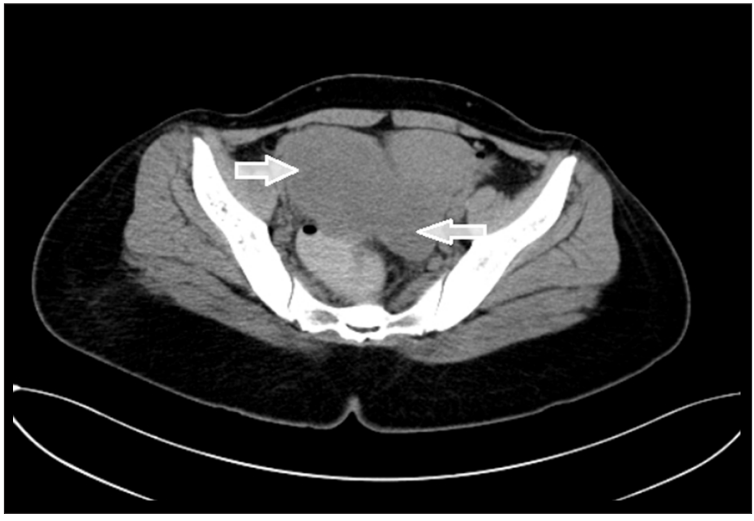

Figure 3. Plain axial CT image of the pelvis shows bilateral ovarian masses (arrows).

on the gradient echo (GRE) sequence due to hemorrhage (Figure 9). Avid peripheral enhancement of both lesions was seen on post-gadolinium enhanced images (Figure 10). The uterus was separately visualized and appeared normal. Fat planes with adjacent structures were preserved. The chest radiograph, that was performed to evaluate pulmonary metastases, was unremarkable.

Subsequently, the patient underwent abdominal hysterectomy with bilateral salpingo-oophorectomy. On gross examination, both ovaries appeared bulky with few hemorrhagic cystic spaces (Figure 11). Microscopic examination was confirmatory for choriocarcinoma, in which blood spaces were surrounded by cytotrophoblast cells that in

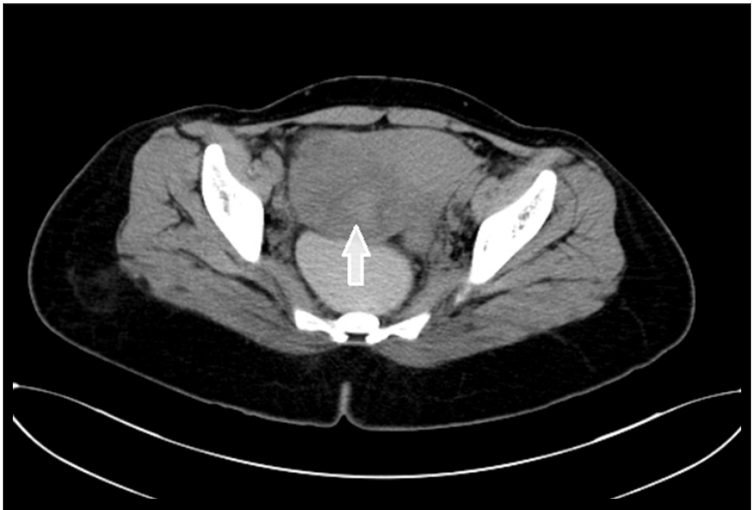

Figure 4. Plain axial CT image at a slightly higher level shows hemorrhage in the right lesion (arrow).

turn were surrounded by syncytiotrophoblast cells. Also, abnormal mitoses with nuclear pleomorphism and hyperchromasia were noted within the cells due to malignancy (Figure 12). No chorionic villi were seen in the specimen. The tumor was positive for $\beta$-HCG on immunohistochemistry. The patient completed a three-month course of chemotherapy and is currently symptom-free with $\beta$-HCG levels within normal limits.

\section{Discussion}

Ovarian choriocarcinoma is an extremely rare subtype of germ cell tumor, first described in 2004 [1]. Less than 1\% of ovarian tumors are choriocarcinomas. Only around thirty 


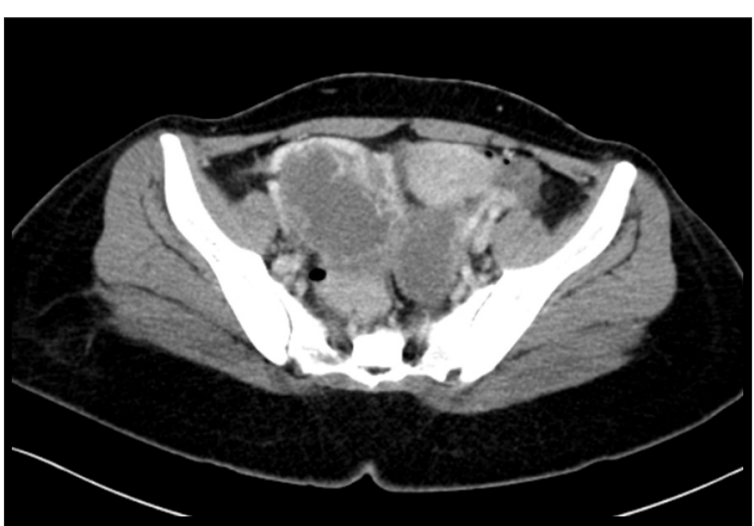

Figure 5. Contrast-enhanced CT image at same level as in Figure 3 shows heterogeneous enhancement of the solid portions of the lesions.

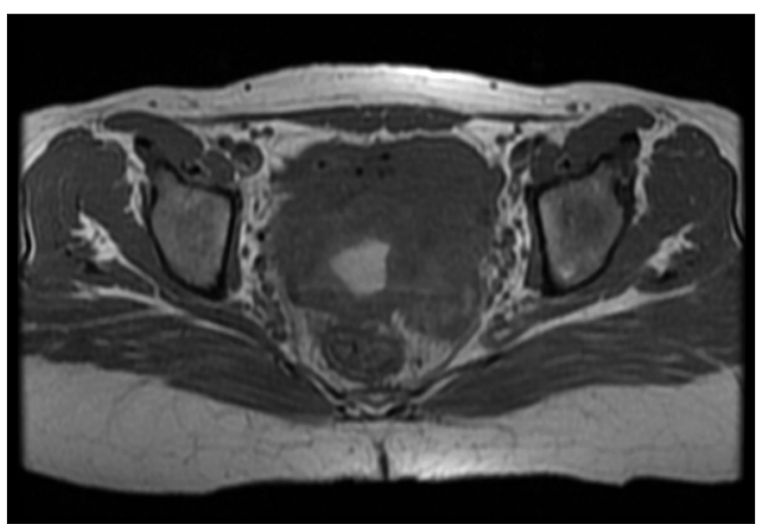

Figure 6. Axial T1W image of the pelvis shows slightly hyperdense bilateral ovarian masses with few areas of hemorrhage.

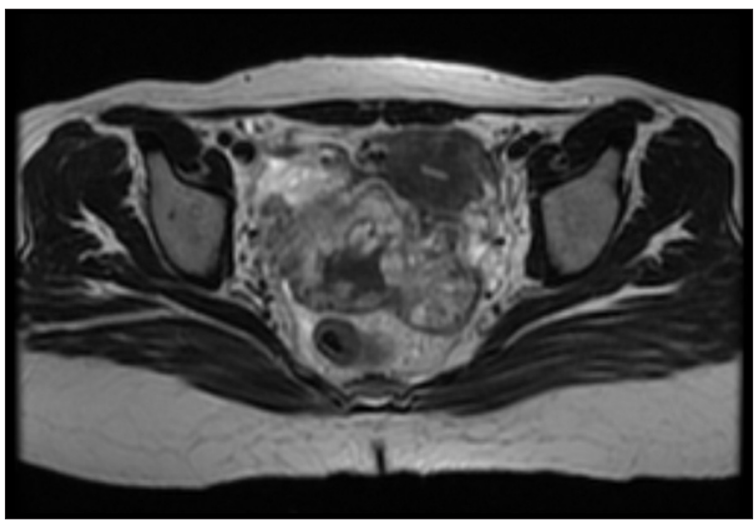

Figure 7. Axial T2W image at same level as in Figure 6 shows hyperintense signal of the masses with central hemorrhage.

cases have been described worldwide to date. Pure primary ovarian choriocarcinoma can arise from old ovarian ectopic pregnancy (gestational) or de novo without any antecedent inciting cause (non-gestational) [2]. Gestational choriocarcinoma can be distinguished from the non-gestational type by DNA polymorphism analysis, whereby presence of paternal antigens in the specimen suggests the gestational type [3]. However, it is a highly expensive test and could not be performed in our department. On ultrasound imaging, choriocarcinomas appear as solid adnexal masses with cystic areas containing dense mobile echoes and septations

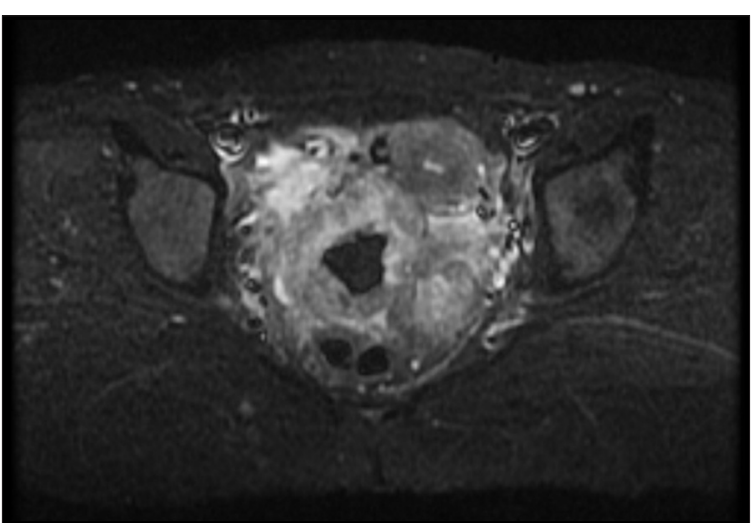

Figure 8. Axial STIR image at same level as in Figure 7 shows lesions to have peripheral high-signal intensity with central low signal.

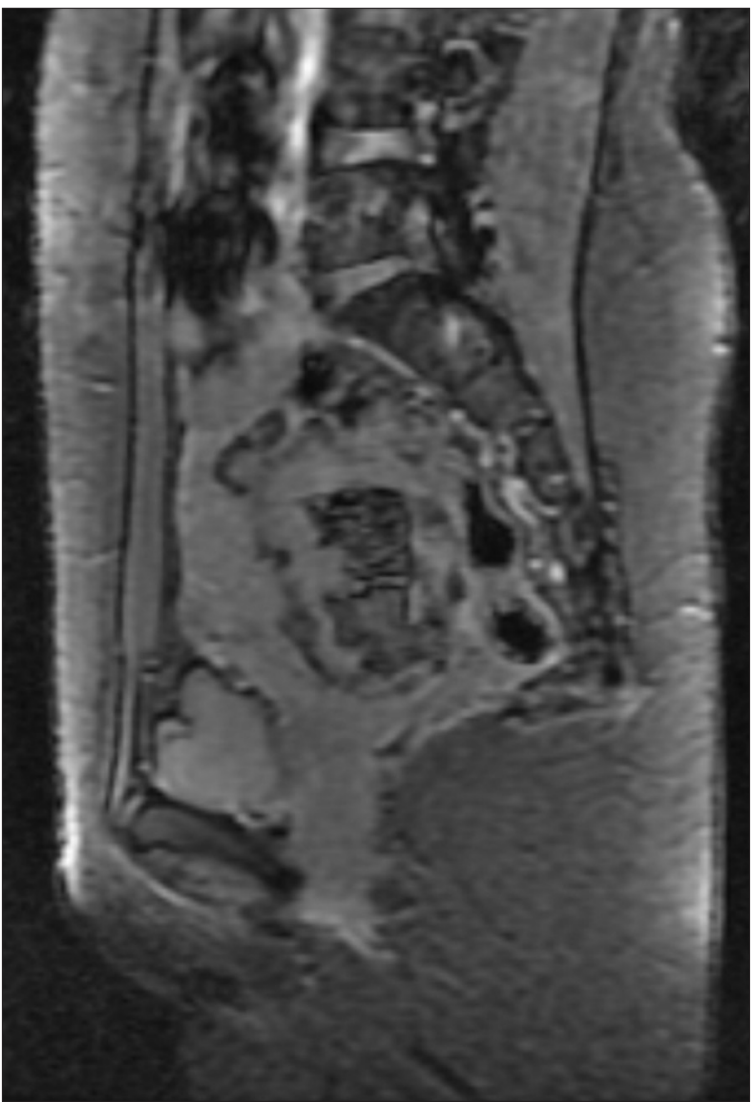

Figure 9. Sagittal oblique GRE image shows central area of blooming within the ovarian mass.

due to hemorrhage. Intense vascularity with low-resistance arterial waveforms can be seen on color Doppler. On CT, choriocarcinoma appears as a well-defined, lobulated, hypodense mass with areas of hemorrhage. Intense enhancement is seen on post-contrast images. Prominent ovarian and uterine vessels are also seen, signifying the vascular nature of choriocarcinoma. MRI, with its excellent soft tissue resolution, shows these masses as iso-tohyperintense lesions on $\mathrm{T} 1, \mathrm{~T} 2$, and STIR images, with avid enhancement of the solid portion of the tumor. Areas of hemorrhage appear as hyperintensities on TlW sequences and as blooming on GRE images. Gross surgical specimens 


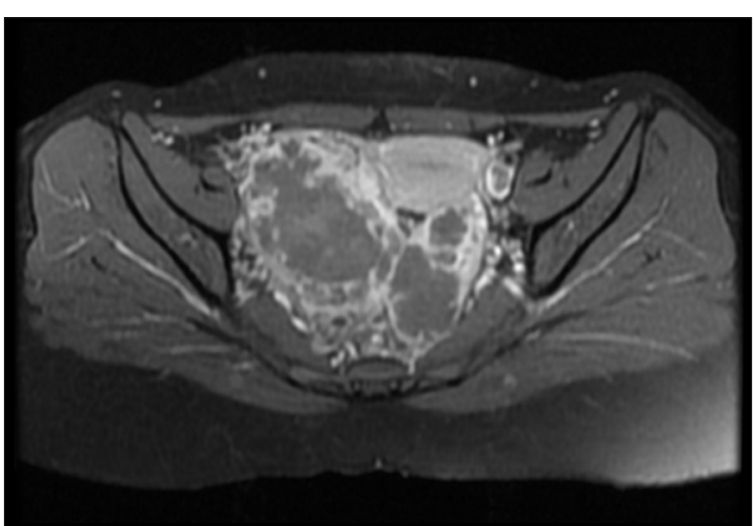

Figure 10. Axial fat-saturated post-contrast T1W images show avid enhancement of both ovarian lesions.

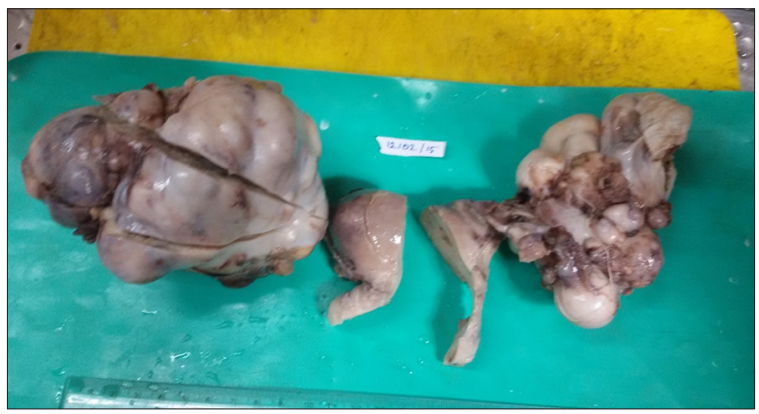

Figure 11. Gross surgical specimen shows bulky ovaries with cystic and hemorrhagic spaces (arrow).

appear as solid masses with blood-filled spaces. The presence of trophoblastic cells and blood spaces with cellular atypia and nuclear polymorphism is specific for choriocarcinoma on the microscopic examination. Absence of intrauterine trophoblastic tissue can aid in the diagnosis of the non-gestational subtype of primary ovarian choriocarcinoma. Although both gestational and non-gestational types secrete excess $\beta$-HCG, the levels of $\beta$-HCG are relatively lower in the non-gestational type [4].

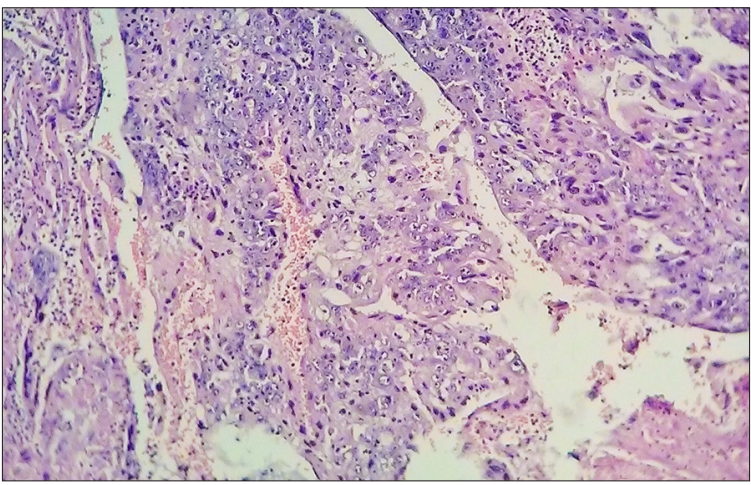

Figure 12. High-power microscopic histopathological specimen shows syncytiotrophoblast and cytotrophoblast cells with nuclear pleomorphism and increased mitoses.

The main differential diagnoses of ovarian choriocarcinoma are other germ cell tumors of the ovary, ectopic pregnancy and tubo-ovarian masses due to chronic infection [5]. Treatment of ovarian choriocarcinoma is either chemotherapy, surgery, or a combination of the two $[6,7]$. However, treatment is dependent on the subtype of choriocarcinoma [8]. The gestational subtype has been shown to respond better to chemotherapy [9], particularly to bleomycin, etoposide, and cisplatin $[10,11]$. Cases treated successfully with chemotherapy show reduction in lesion size and $\beta$-HCG levels.

\section{Conclusions}

Based on clinical history, imaging findings, and serological and histopathological evidence, a final diagnosis of bilateral primary non-gestational choriocarcinoma was made, and the patient was managed accordingly.

\section{Acknowledgement}

We would like to express our heartfelt gratitude to Dr. Hadiya Naureen from Department of Pathology, Bapuji Hospital for her selfless help in this article.

\section{References:}

1. Bazot M, Cortez A, Sananes S, Buy J-N: Imaging of pure primary ovarian choriocarcinoma. Am J Roentgenol, 2004; 182: 1603-4

2. Talerman A. Germ cell tumors. Curr Top Pathol. 1992;85: 165-202

3. Koo HL, Choi J, Kim KR, Kim JH: Pure non-gestational choriocarcinoma of the ovary diagnosed by DNA polymorphism analysis. Pathol Int, 2006; 56(1): 613-16

4. Mood NI, Samadi N, Rahimi-Moghaddam P et al: Pure ovarian choriocarcinoma: Report of two cases. J Res Med Sci, 2009; 14(5): 327-30

5. Balat O, Kutlar I, Ozkur A et al: Primary pure ovarian choriocarcinoma mimicking ectopic pregnancy: A report of fulminant progression. Tumori, 2004; 90: 136-38

6. Gershenson DM: Management of early ovarian cancer: Germ cell and sex cord-stromal tumors. Gynecol Oncol, 1994; 55: S62-72
7. Shanmugasundarama G, Sundaramoorthya E, Suresh Sudalaiandi $S$ et al: Double pathology: Malignant epithelial ovarian tumor and germ cell tumor (choriocarcinoma), a rare coexistence. World $J$ Oncol, 2015; 6(4): 421-25

8. Errarhay S, Hmidani N, Mahmoud S et al: Rare tumor of the ovary: Primitive choriocarcinoma. Open Journal of Obstetrics and Gynecology, 2013; 3: 448-50

9. Lorigan PC, Colman RE, Hancock BW: The treatments of persistent trophoblastic disease using the Sheffield modification of chaing cross risk score. Proc Annu Meeting Am Soc Clin Oncol, 1994; 13: 257

10. Lv L, Yang $\mathrm{K}, \mathrm{Wu} \mathrm{H}$ et al: Pure choriocarcinoma of the ovary: A case report. J Gynecol Oncol, 2011; 22(2): 135-39

11. Hayashi S, Abe Y, Tomita S et al: Primary non-gestational pure choriocarcinoma arising in the ovary: A case report and literature review. Oncol Lett, 2015; 9: 2109-11 